\title{
Depresión y Bullying en adolescente del Liceo María Teresa Angulo extensión Bojo Sanare Estado Lara
}

\section{Depression and Bullying in adolescent of the Liceo María Teresa Angulo extension Bojo Sanare Estado Lara}

\author{
Ana Jiménez \\ mavafe30@gmail.com \\ Universidad Nacional Experimental Francisco de Miranda \\ Venezuela \\ Doralys Silva \\ cienciamatria@gmail.com \\ Universidad Nacional Experimental Francisco de Miranda \\ Venezuela \\ Noheli Ortiz \\ cienciamatria@gmail.com \\ Universidad Nacional Experimental Francisco de Miranda \\ Venezuela \\ Marinaisabel Vargas \\ cienciamatria@gmail.com \\ Universidad Nacional Experimental Francisco de Miranda \\ Venezuela
}

Recibido: $17 / 06 / 2017$

Aprobado: 23/07/2017 


\section{RESUMEN}

El propósito de la investigación fue determinar los niveles de depresión en Adolescentes Escolarizados implicados en la dinámica Bullying en el liceo María Teresa Angulo extensión Bojo Barquisimeto Sanare Estado Lara, periodo Enero-Junio 2017.Es una investigación de tipo Descriptiva, no experimental, donde la población estuvo constituida por 50 adolescentes voluntarios a quienes se les aplicó el cuestionario para la exploración del bullying (CEBU). Posteriormente a los alumnos implicados en la dinámica bullying se les aplicó el instrumento de Beck para evaluar niveles de depresión. De 50 alumnos, 28 resultaron implicados en Bullying, 11 alumnos fueron identificados como fuertes (40\%); 4 alumnos además de fuertes son provocadores (14\%); 3 alumnos son clasificados provocadores y Agresivo (11\%); 2 alumnos es Agresivo y Provocador (7\%). Igualmente, 3 alumnos sus compañeros manifiestan tenerle idea (11\%), 3 alumnos son considerados por sus compañeros como cobarde (11\%); 1 alumno es visto como cobardes-víctimas (3\%),) y un alumno que además de víctima es catalogada como provocador (3\%). De los alumnos implicados en la dinámica bullying el $50 \%$ de la muestra presento ausencia de depresión, $32 \%$ presento sintomatología depresiva no incapacitante y un $11 \%$ presentaron depresión moderada y un $7 \%$ presento depresión severa.

Palabras claves: Adolescentes, Bullying, víctimas, hostigamiento, depresión.

\section{ABSTRACT}

The purpose of the research was determine the levels of depression in Adolescents Schooling involved in the dynamics Bullying in the high school Maria Teresa Angulo extension Bojo Barquisimeto Sanare Edo Lara, period January-June 2017 . Descriptive, non-experimental research, where the population consisted of 50 volunteer adolescents, who were administered the questionnaire exploration Bullying (CEBU), Later students involved I were applied instrument beck to assess depression. Of a total of 50 students, 28 students were involved in Bullying. 11 students were identified as strong (40\%); 4 students as well as strong are provocative (14\%); 3 students are classified as aggressive and Aggressive (11\%); 2 students are Aggressive and Provocative (7\%). Likewise, 3 students (11\%) say they have an idea (11\%), 3 students are considered by their peers as a coward (11\%); 1 pupil is seen as coward-victims (3\%), and a pupil who, in addition to being a victim, is classified as a provocateur (3\%). of the students involved in the dynamic bullying, the depressive symptomatology was absent, $50 \%$ of the sample studied), 32\% mild depression, $11 \%$ moderate depression (7\%) severe depression. 


\section{CIENCIAMATRIA \\ Revista Interdisciplinaria de Humanidades, Educación, Ciencia y Tecnología \\ Año V. Vol. V. №9. Julio - Diciembre 2019 \\ Hecho el depósito de ley: pp201602FA4721 \\ ISSN-L: 2542-3029; ISSN: 2610-802X \\ Universidad Nacional Experimental Francisco de Miranda (UNEFM). Santa Ana de Coro. Venezuela}

Ana Jiménez; Doralys Silva; Noheli Ortiz; Marinaisabel Vargas

Keywords: Teenagers, Bullying, victims, harassment, Depression.

\section{INTRODUCCIÓN}

La adolescencia es una etapa trascendental para el desarrollo humano, debido a la serie de cambios fisiológicos y psicológicos e interpersonales que conlleva. Dichos cambios hacen al adolescente vulnerable ya que surge una crisis de identidad que los enfrenta a sí mismos, a las demandas familiares y de la sociedad produciéndoles gran ansiedad, rabia y confusión. Sentimientos que en ocasiones el adolescente no puede afrontar debido a diferentes factores, entre ellos sus características de personalidad, el estrés cotidiano y la ausencia de redes de apoyo sociales y familiares.

Por consiguiente, esos factores favorecen la presencia de malestar emocional en el adolescente, el cual puede desembocar en conductas de riesgos como son el consumo de drogas e intento de suicidio, que comprometen su integridad física y psicológica. Ponce Malaver Moisés (2015) Es una etapa de la vida humana en que “... Los procesos psicológicos del individuo y las pautas de identificación evolucionan desde los del niño a las del adulto... ". En los casos de la adolescencia la integración social es relevante para el normal desarrollo y para la educación de los jóvenes entonces, es allí donde las instituciones educativas deben fomentar un clima de tolerancia, valores que normalice la percepción de las diferencias entre los adolescentes.

En efecto, la intimidación es otros de los factores que afecta a los jóvenes de todas las razas y clases, y es motivo de preocupación para mucho, no solamente para quienes son víctimas de ella; pero, debido a que los padres, los maestros y otros adultos no siempre pueden presenciarla, muchas veces no entienden hasta qué extremo puede llegar a afectarlos.

Ahora bien, el Bullying no es tema nuevo en nuestra sociedad más sin embargo, es un tema que últimamente ha tenido mayor impacto debido a la cantidad de casos de persecución y de agresión que se han detectado en las escuelas, que han llevado a los 


\section{CIENCIAMATRIA \\ Revista Interdisciplinaria de Humanidades, Educación, Ciencia y Tecnología \\ Año V. Vol. V. №9. Julio - Diciembre 2019 \\ Hecho el depósito de ley: pp201602FA4721 \\ ISSN-L: 2542-3029; ISSN: 2610-802X \\ Universidad Nacional Experimental Francisco de Miranda (UNEFM). Santa Ana de Coro. Venezuela}

Ana Jiménez; Doralys Silva; Noheli Ortiz; Marinaisabel Vargas

agredidos a situaciones realmente aterradoras. El acoso escolar (Bullying en inglés) Gómez Nashiki 2013 confirma "se constituye una práctica más generalizada a nivel mundial, esto hace parte a las preocupaciones de padres, docente, psicólogos y otros especialistas que intentan encontrar una respuesta que permita la intervención y la orientación.

Dentro de esta perspectiva, según una publicación de La Organización Mundial de la Salud (OMS) en el 2010, para las autoridades sanitarias de los Estados Unidos el bullying se ha convertido en una crisis, ya que la tasa de suicidio consumado en escolares y adolescentes se ha incrementado en los últimos 5 años de un 8,9\% a un $16 \%$, de los cuales más del $58 \%$ están asociados a acoso escolar entre pares.

Por consiguiente, los estudiantes involucrados en acoso escolar tienen un alto riesgo de experimentar un amplio espectro de manifestaciones psicosomáticas, tales como ausencia del hogar, ausentismo escolar, abuso de drogas y alcohol, daños físicos infligidos por otros o auto infligidos, accidentes y hasta el suicidio, reportándose a nivel mundial múltiples casos de muertes asociadas a bullying $(5,6,7)$. Desde la perspectiva más general, este problema se presenta en múltiples formas; pero con características específicas; debe darse entre pares de la misma edad y se trata de una exposición repetitiva de una persona a la agresión física y/o emocional incluyendo sobrenombres, burlas, muecas, manotones, exclusión social, malos tratos y rumores.

Los individuos pueden ser bullies, perpetradores o agresores, víctimas o incluso ambas (9). En el Bullying a diferencia de otros tipos de violencia, no media una provocación por parte de la víctima, además existe un desequilibrio de poder entre los participantes, siempre se presentan todos los elementos de manera conjunta y las razones por las que este problema se presenta de una u otra manera aún no han sido identificadas (9).

Es así como, en un reciente estudio realizado por García X y col., de una muestra representativa de 2.727 estudiantes de 66 centros escolares de secundaria en Barcelona, España. Se definió el "acoso escolar" como haber sido objeto de burla, 


\section{CIENCIAMATRIA \\ Revista Interdisciplinaria de Humanidades, Educación, Ciencia y Tecnología \\ Año V. Vol. V. №9. Julio - Diciembre 2019 \\ Hecho el depósito de ley: pp201602FA4721 \\ ISSN-L: 2542-3029; ISSN: 2610-802X \\ Universidad Nacional Experimental Francisco de Miranda (UNEFM). Santa Ana de Coro. Venezuela}

Ana Jiménez; Doralys Silva; Noheli Ortiz; Marinaisabel Vargas

golpeado o marginado cuatro o más veces. Se realizó un análisis de regresión logística bivariado y multivariado para estudiar la relación entre el acoso y diversos factores, incluyendo variables socio demográficas, actitudes y comportamientos.

La prevalencia de acoso escolar fue del $18,2 \%, 10,9 \%$ y $4,3 \%$ en chicos, y del $14,4 \%$, $8,5 \%$ y $4,5 \%$ en chicas de $2^{\circ}$ y $4^{\circ}$ de educación secundaria obligatoria y $2^{\circ}$ de bachillerato o ciclos formativos de grado medio, respectivamente. Los factores que se asociaron con un incremento de la probabilidad de padecer acoso fueron el estado de ánimo negativo y la conducta violenta, mientras que tener mayor edad implica un riesgo mayor en la medida que el individuo se expone a situaciones de consumo de alcohol, drogas, asistir a sitios como bares y discotecas entre otros (10).

En este mismo orden de ideas, en Latinoamérica, Romaní $\mathrm{F}$ y Gutiérrez $\mathrm{C}$, realizaron un análisis secundario de los datos del II Estudio Nacional de Prevención de violencia escolar en Perú, donde participaron 526 colegios entrevistando a 65.041 estudiantes entre 11 y 19 años de ciudades de la Costa, Sierra y Selva, con una tasa de rechazo de $9,1 \%$, usando una encuesta auto aplicada.

Evidenciando una prevalencia de victimización del 56,4\% y frecuencia de victimización severa de un $8,9 \%$, siendo estas cifras muy elevadas, mayor que las reportadas en otros países sudamericanos y Europa (11). La Organización No Gubernamental ONG) Bullying Sin Fronteras (2015) junto a su fundador el Dr. Javier Miglino alertaron que sigue creciendo el acoso en la escuela o Bullying en el ámbito de la República Argentina, manteniéndose el crecimiento de la problemática en un 40 por ciento con relación al año anterior. Desde el año 2013 junto al equipo interdisciplinario de Bullying Sin Fronteras, conformado por médicos, psiquiatras, psicólogos, psicopedagogos, educadores, abogados, periodistas y padres de chicos que han padecido Bullying.

De este modo, el Informe Nacional de Bullying en ese país demostró que permanentemente las cifras crecen y se tienen más casos denunciados, estableciéndose que hay más violencia en los chicos. Miglino (2016) dijo "Ya no solo se 


\section{CIENCIAMATRIA \\ Revista Interdisciplinaria de Humanidades, Educación, Ciencia y Tecnología \\ Año V. Vol. V. No9. Julio - Diciembre 2019 \\ Hecho el depósito de ley: pp201602FA4721 \\ ISSN-L: 2542-3029; ISSN: 2610-802X \\ Universidad Nacional Experimental Francisco de Miranda (UNEFM). Santa Ana de Coro. Venezuela}

Ana Jiménez; Doralys Silva; Noheli Ortiz; Marinaisabel Vargas

padece Bullying por el mayor rendimiento escolar o atributos físicos sino directamente la belleza en particular de las chicas las ha hecho foco de golpes, amenazas y burlas y los viajes a Bariloche que antes eran momentos de esparcimiento y alegría con vistas a la finalización de los estudios cada vez se parecen más a las "novatadas universitarias" que se practican desde hace años con resultados violentos en universidades de los Estados Unidos de América de parte de estudiantes avanzados sobre los recién llegados".

De igual manera, informó Miglino (2016) que para el año 2015 finalizó con 1.631 casos graves de bullying pero el primer semestre de concluyó con 1.142 casos, lo que anualizado nos da un crecimiento del 40 por ciento de casos graves de acoso escolar denunciados en la justicia y los ministerios de educación de la República Argentina. Más de la mitad de ellos en Capital Federal, el Conurbano bonaerense, Córdoba, Mendoza, San Luis, San Juan, Formosa, Corrientes y Misiones.

En México, según cifras de la Comisión Nacional de los Derechos Humanos (CNDH) (2011) el bullying es un problema que se extiende donde el número de casos aumentó en $10 \%$ durante los últimos dos años (13). La CNDH reportó que el $30 \%$ de los estudiantes de primaria declaraba sufrir algún tipo de Bullying. Para 2013, el porcentaje subió al $40 \%$. Con esta cifra, según la Organización para la Cooperación y el Desarrollo Económicos (OCDE), México llegó al primer lugar internacional en este problema, pues 18 millones 781,875 alumnos de primaria y secundaria, tanto en escuelas públicas como privadas, sufren acoso escolar.

En el caso de Venezuela, de acuerdo al informe del Centro Comunitario de Aprendizaje por los derechos de la niñez y adolescencia (CECODAP) en conjunto con la Universidad Católica Andrés Bello, se realizó un estudio en 32 instituciones educativas de Caracas, reportó que un 9\% de la población escolar ha sufrido intimidación (9). Los niños, niñas y adolescentes están expuestos a diversas situaciones de violencia, sin 


\section{CIENCIAMATRIA \\ Revista Interdisciplinaria de Humanidades, Educación, Ciencia y Tecnología \\ Año V. Vol. V. №9. Julio - Diciembre 2019 \\ Hecho el depósito de ley: pp201602FA4721 \\ ISSN-L: 2542-3029; ISSN: 2610-802X \\ Universidad Nacional Experimental Francisco de Miranda (UNEFM). Santa Ana de Coro. Venezuela}

Ana Jiménez; Doralys Silva; Noheli Ortiz; Marinaisabel Vargas

embargo, se mantienen la violencia social y la escolar como más frecuentes (violencia social: $39,26 \%$, violencia escolar: $25,89 \%)$.

Por su parte, sólo unos pocos estudios poblacionales han examinado prospectivamente los efectos del acoso. Bond y col., en una muestra de 2.680 estudiantes, en una escuela de Australia, demostraron que la persecución a los 8 y 9 años de edad, predispuso a la existencia de síntomas auto notificados de ansiedad y depresión al cabo de hasta 3 años (15). Del mismo modo la Dra. Arsenault y col., observaron que las víctimas y los agresores/víctimas presentaron más problemas del comportamiento y de ajuste escolar a los siete años de edad, incluso tras el control respecto a problemas previos de ajuste a los cinco años de edad, en un estudio de cohorte representativo nacionalmente con una muestra de 2.232 niños de 5 a 7 años, en Londres.

Además, evaluaron si la victimización e intimidación frecuente en la infancia aumentaba las posibilidades de dañarse a sí mismos en la adolescencia temprana. Entre los niños de 12 años de edad que se habían hecho daño auto infligido $(2,9 \%, n=62)$, más de la mitad fueron víctimas de acoso frecuente $(56 \%, n=35)$. La exposición frecuente a intimidación predijo mayores tasas de autolesiones, incluso años después de haber sido reportadas. En comparación con los niños intimidados que no se hicieron daño a sí mismos y los intimidados que se autolesionaron se evidenciaron antecedentes familiares de intento de suicidio / suicidio consumado, problemas de salud mentales recurrentes y una historia de maltrato físico por parte de un adulto.

A este propósito, los investigadores del Hospital Psiquiátrico Infantil Dr. Juan Navarro de México, Albores- Gallo y col., realizaron un estudio con la finalidad de definir la relación entre el tipo de psicopatología presentada por los escolares y el grado de acoso escolar, en una muestra de niños de las escuelas públicas de la Ciudad de México, donde participaron un total de 1092 escolares; identificando mediante el Test Bull-S a un grupo de agresores y neutros; 23,9 \% estuvo implicado en el fenómeno de 


\section{CIENCIAMATRIA \\ Revista Interdisciplinaria de Humanidades, Educación, Ciencia y Tecnología \\ Año V. Vol. V. №9. Julio - Diciembre 2019 \\ Hecho el depósito de ley: pp201602FA4721 \\ ISSN-L: 2542-3029; ISSN: 2610-802X \\ Universidad Nacional Experimental Francisco de Miranda (UNEFM). Santa Ana de Coro. Venezuela}

Ana Jiménez; Doralys Silva; Noheli Ortiz; Marinaisabel Vargas

acoso escolar con una media de edad de 9,6 años clasificados de la siguiente manera $8,97 \%$ agresores, $12,27 \%$ víctimas, $2,7 \%$ víctimas y agresores y $76 \%$ controles.

Es decir, que el grupo de agresores tuvo asociación con las escalas de ansiedad, síntomas somáticos, oposicionismo y de conducta. Mientras que en el grupo de víctimas las asociaciones encontradas fueron problemas de ansiedad. Por lo que el acoso escolar en dicho estudio se asocia con psicopatología que requiere atención psiquiátrica oportuna.

De todas las conductas violentas en la escuela, las peleas entre compañeros son las más frecuentes, bien entre iguales o entre pandillas. Pero sin duda alguna, el acoso escolar o Bullying y su presencia en las escuelas supone para las víctimas según Dan Olweus (Ob. Cit)"una conducta de persecución física y psicológica que realiza un alumno hacia otro, el cual es elegido como víctima de repetidos ataques." Esta acción, negativa e intencionada, sitúa a las víctimas en posiciones de las que difícilmente pueden salir por sus propios medios. Se evidencia claramente un desbalance de poder que se ejerce en forma intimidatoria al más débil y por tanto escogido, no al azar, con la intención premeditada de causar daño, repetido en el tiempo.

Ahora bien, la relación entre el Bullying y la depresión se da cuando la victimización está asociada al aislamiento y de igual manera a una menor valoración de parte de sus compañeros; también se puede presentar un rendimiento académico bastante bajo y se expresa por medio de los sentimientos de tristeza, ya que no se sienten seguros porque no tienen sentido de pertenencia en el colegio. Galdó, Redondo y García (2008) comentan respecto a las consecuencias psicológicas en las víctimas de Bullying que están: el Aislamiento y Síntomas Somáticos. En el primero las víctimas suelen presentar en sus patologías sentimientos de tipo depresivo y de soledad, pero es bastante evidente que se presente ansiedad 0 baja autoestima, y sufren un considerable distrés. 


\section{CIENCIAMATRIA \\ Revista Interdisciplinaria de Humanidades, Educación, Ciencia y Tecnología \\ Año V. Vol. V. No9. Julio - Diciembre 2019 \\ Hecho el depósito de ley: pp201602FA4721 \\ ISSN-L: 2542-3029; ISSN: 2610-802X \\ Universidad Nacional Experimental Francisco de Miranda (UNEFM). Santa Ana de Coro. Venezuela}

Ana Jiménez; Doralys Silva; Noheli Ortiz; Marinaisabel Vargas

Por lo tanto, cuando se asocia Bullying y la depresión los síntomas somáticos se puede observar una sintomatología como las cefaleas, dolores de estómago, espalda, mareos, cansancio, dificultad para dormir y expresan con mayor frecuencia ideación autolítica y más conductas de suicidio De acuerdo a Oñerreda 2008 las víctimas sufren de varias consecuencias entre las cuales están el bajo rendimiento académico, el fracaso escolar, la ansiedad, la depresión, los sentimientos de culpa, el pobre autoconcepto. La carencia de asertividad, la inseguridad que presentan los niños o niñas ante lo que realizan en clase, ya que no están seguros de lo que ellos creen, así mismo sufren de baja popularidad; también se puede observar las pocas relaciones sociales con las que estos cuentan ya que no tienen contacto con sus amigos.

Es por ello, que McDowell y Hostetle 2010, describen que la depresión en los jóvenes es difícil de detectar debido a que sus síntomas son diferentes a los clásicos de la depresión en un adulto, esto se debe a que el adolescente sabe disimularla. También se puede decir que se dificulta ya que se puede llegar a confundir o puede ir acompañado de otras cosas como lo son el síndrome premenstrual en las jóvenes. Los jóvenes no necesitan una razón de peso para deprimirse ya que son vulnerables a las situaciones que viven en el ambiente y la sensibilidad de estos se altera por el manejo de las emociones en conflicto junto con el querer saber de la sexualidad, ya que los cambios que 18 ocurren en el cuerpo del adolescente no son similares.

Considerando la información previamente expuesta, el presente trabajo es importante porque busca identificar la Depresión en Adolescente involucrados en la dinámica de Bullying en Bojo Sanare Edo Lara (periodo 2016-2017),

A continuación se citan algunos estudios que tiene relación directa con la investigación: Monelos (2015) Universidad de Coruña realizó un estudio empírico desde un punto de vista descriptivo y exploratorio, con el objetivo de analizar el Bullying entendido como una forma específica de violencia escolar, donde uno o varios agresores con mayor poder y con intencionalidad de causar dolor acosan y someten reiteradamente a un 


\section{CIENCIAMATRIA \\ Revista Interdisciplinaria de Humanidades, Educación, Ciencia y Tecnología \\ Año V. Vol. V. №9. Julio - Diciembre 2019 \\ Hecho el depósito de ley: pp201602FA4721 \\ ISSN-L: 2542-3029; ISSN: 2610-802X \\ Universidad Nacional Experimental Francisco de Miranda (UNEFM). Santa Ana de Coro. Venezuela}

Ana Jiménez; Doralys Silva; Noheli Ortiz; Marinaisabel Vargas

compañero/a, tal como ha quedado de manifiesto. Se concluyen que los alumnos de $1^{\circ}$ y $2^{\circ}$ de la ESO cuando les ocurre acoso escolar en el colegio un $33,69 \%$ no comunican la situación y cuando se comunica la situación de acoso escolar, un $62 \%$ se lo transmite al profesorado y un $73,5 \%$ se lo comunica a la familia.

Por otra parte, Mejía (2011) expuso sobre El Bullying: el caso de la facultad de Contaduría y Administración de la Universidad Veracruzana región Xalapa. Los resultaos obtenidos en cuanto a los victimarios es impactante confesaron sus acciones, y quizás esto se debe a que para ellos el empujar a alguien, esconder un objeto personal, el poner apodos, el burlarse de alguien, etc. no tiene gran importancia o valor para el victimario y solo lo ven como un juego.

También, en el contexto nacional Acosta Rubí y Moreno Lismayka (2015) presentaron el trabajo de grado en la Universidad de Carabobo, titulado Estrategias de Convivencia Escolar para la Prevención del Bullying en la Escuela Técnica "Los Magallanes" del Municipio San Diego del Estado Carabobo. Este trabajo de investigación tuvo por objetivo Diseñar una estrategia de convivencia escolar para la prevención del bullying en la Escuela Técnica "Los Magallanes" Los resultados muestran que en el centro tienen lugar todos los tipos de acoso, existiendo una relación inversa entre la "gravedad" de la conducta de acoso y la "frecuencia" de agresiones verbales, exclusión social y la agresión física indirecta son las formas de acoso más frecuentes, siendo las aulas de clase, cancha o baños los escenarios elegidos para estas conductas agresivas. (34).

Asimismo, Díaz, Vanessa Daniella (2009) en presentó el trabajo de grado titulado "Síndrome Bullying en estudiantes del ciclo diversificado del Liceo Bolivariano "Fernando Peñalver" de Ciudad Bolívar. Abril - Julio que se basó en demostrar la existencia del síndrome Bullying en dicha institución, así como las causas, escenarios más frecuentes, formas de maltrato y actitud del profesorado para intervenir en los 


\section{CIENCIAMATRIA \\ Revista Interdisciplinaria de Humanidades, Educación, Ciencia y Tecnología \\ Año V. Vol. V. №9. Julio - Diciembre 2019 \\ Hecho el depósito de ley: pp201602FA4721 \\ ISSN-L: 2542-3029; ISSN: 2610-802X \\ Universidad Nacional Experimental Francisco de Miranda (UNEFM). Santa Ana de Coro. Venezuela}

Ana Jiménez; Doralys Silva; Noheli Ortiz; Marinaisabel Vargas

hechos de violencia en dicho plantel, mediante un estudio descriptivo de campo, analítico y de tipo transversal.

Los datos obtenidos mediante el cuestionario de Pre concepciones de intimidación y maltrato entre iguales (PRECONCIMEI), adaptado de Ortega, Mora - Merchán y Mora, para el alumnado y profesores, obteniendo que la forma más frecuente de maltrato es insultar y poner motes $(29,1 \%)$. Se determinó que los escenarios de intimidación más frecuentes son la calle $(25,4 \%)$ y cerca del instituto al salir de clase $(21,7 \%)$. En relación a la causa más frecuente de maltrato entre iguales se encontró que lo hacían por molestar $(41,8 \%)$.

Con respecto a los protagonistas de este estudio resultó un total de 129 agresores, de los cuales el sexo predominante fue masculino $(54,4 \%)$, y el grupo etario que prevaleció fue de $16-17$ años $(69,8 \%), 103$ víctimas, predominando el sexo femenino (67\%) y el grupo etario entre $16-17$ años (67\%) y 187 observadores, siendo mayor la muestra femenina $(57,8 \%)$ y el rango etario entre los $16-17$ años $(74,3 \%)$; las cifras obtenidas en la investigación muestran la presencia de Bullying en la población estudiantil de la Institución.(35)

Estos hechos dan la iniciativa para justificar la presente investigación porque los padres y los maestros deben ser conscientes de esta problemática que conduce a los peligros a largo plazo de la intimidación por lo que es necesario detenerlo donde empieza. Es un asunto social y requiere la participación de todos los actores, las experiencias escolares más allá de los asuntos académicos es realmente importante, y se necesita diseñar intervenciones que sean capaces de contrarrestar la victimización del acoso, conflicto y frustración. Particularmente durante el desarrollo del adolescente, cuando las relaciones entre compañeros son de suma importancia, los eventos traumáticos relacionados con las relaciones entre compañeros, tales como ser intimidado, podrían causar trauma lo suficientemente grave como para conducir a la depresión. 


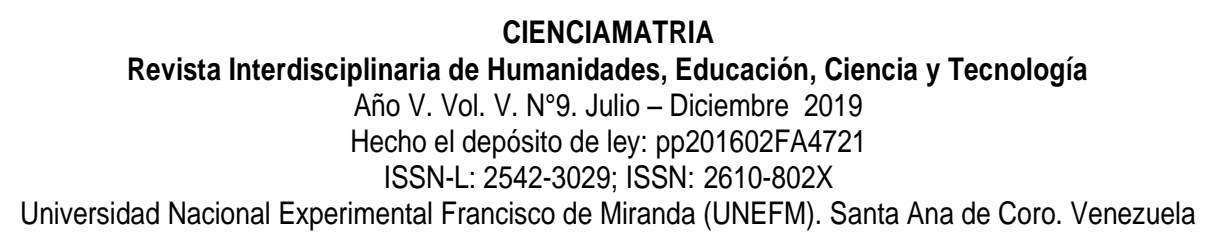

Ana Jiménez; Doralys Silva; Noheli Ortiz; Marinaisabel Vargas

Aunque el problema del acoso entre alumnos ha tenido recientemente gran importancia en la sociedad, su abordaje se realiza en la mayoría de los casos desde una óptica puramente escolar, sin prestar atención a las repercusiones psicológicas para los involucrados, la presente investigación pretende Evaluar la depresión en Adolescente Escolarizados implicados en dinámica Bullying en el liceo María Teresa Angulo extensión Bojo, Sanare Edo Lara, periodo Enero-Junio 2017

\section{OBJETIVOS DE LA INVESTIGACIÓN Objetivo general}

Determinar los niveles de depresión en Adolescente Escolarizados implicados en la dinámica Bullying en el liceo María Teresa Angulo extensión Bojo, Sanare Edo Lara, periodo Enero-Junio 2017

\section{Objetivos Específicos}

Describir las características demográficas de los adolescentes. Identificar los adolescentes implicados en la dinámica Bullying.

Evaluar el nivel de depresión de los adolescentes implicados en la dinámica Bullying.

\section{MATERIALES Y METODOS}

Tipo de Investigación descriptiva, con diseño de campo, no experimental, de corte Transversal. La población estuvo representada por un total de 50 adolescentes cursantes de educación media y diversificada del liceo María Teresa Angulo extensión Bojo, Sanare Edo Lara, durante el periodo Enero-Junio 2017. La muestra quedó constituida por 28 adolescentes implicados en la dinámica bullying.

\section{Técnica e instrumento de recolección de datos:}

Para llevar a cabo la investigación en primer lugar se realizó una entrevista con la directora del liceo María Teresa Angulo extensión Bojo, Sanare Edo Lara, con el fin de 


\section{CIENCIAMATRIA \\ Revista Interdisciplinaria de Humanidades, Educación, Ciencia y Tecnología \\ Año V. Vol. V. No9. Julio - Diciembre 2019 \\ Hecho el depósito de ley: pp201602FA4721 \\ ISSN-L: 2542-3029; ISSN: 2610-802X \\ Universidad Nacional Experimental Francisco de Miranda (UNEFM). Santa Ana de Coro. Venezuela}

Ana Jiménez; Doralys Silva; Noheli Ortiz; Marinaisabel Vargas

solicitar el permiso para realizar la investigación y obtener los datos de la población. Posteriormente se realizó una reunión con padres y representantes para obtener la firma del consentimiento informado y explicarles en que consiste la investigación y los beneficios que los adolescentes implicados en la dinámica bullying con depresión serán canalizados a consulta especializada y se le darán las recomendaciones para evitar depresión.

Luego se les aplicó el instrumento realizado por los autores Miguel Ángel Estrada Gómez, y Adia Jak de ipp denominado cuestionario para la exploración del Bullying (CEBU) para evaluar la presencia de Bullying, el cual consta de 70 ítems. Todos ellos con un formato de respuesta de escalamiento tipo Likert de 4 valores numéricos del 1 al 5 donde el 1 es nunca, 2 es casi nunca, 3 es con frecuencia, 4 es casi siempre, y 5 es siempre.

El CEBU está estructurado en 3 partes de acuerdo a los actores principales del Bullying, bulliado (victima), en lo que se contemplan 24 ítems (1 al 24), bullie o bully (agresor), con 24 ítems (25 al 48), y observador que contiene 22 ítems (49 al 70). Posteriormente a los adolescentes implicados en la dinámica Bullying se les aplicara el instrumento de Beck, el cual fue elaborada por Beck, Weissman, Lesster y Trexler, ${ }^{27}$ (1974) para evaluar los niveles de depresión el mismo consta de 21 ítems conteniendo cada uno varias fases auto evaluativas donde el paciente puede seleccionar la que mejor se adapte a su situación; estas afirmaciones reflejaran la severidad del malestar producido por los síntomas depresivos y se calificaran de 0 (mínimo) a 4 (severo).

La puntuación total de la escala se obtendrá sumando los 21 reactivos, siendo 0 la mínima puntuación y 64 la máxima a obtener, estos resultados se interpretaran que de 0 a 9 refleja la ausencia o presencia mínima de síntomas depresivos; de 10 a 16 indica una depresión media; de 17 a 29 refleja una depresión moderada y puntuaciones de 30 a 64 indican una depresión severa. Finalmente se procesaran los datos para elaborar conclusiones y recomendaciones. 


\section{CIENCIAMATRIA \\ Revista Interdisciplinaria de Humanidades, Educación, Ciencia y Tecnología \\ Año V. Vol. V. №9. Julio - Diciembre 2019 \\ Hecho el depósito de ley: pp201602FA4721 \\ ISSN-L: 2542-3029; ISSN: 2610-802X \\ Universidad Nacional Experimental Francisco de Miranda (UNEFM). Santa Ana de Coro. Venezuela}

Ana Jiménez; Doralys Silva; Noheli Ortiz; Marinaisabel Vargas

\section{RESULTADOS}

De los 50 adolescentes que conformaron la muestra en estudio se registró una edad promedio de 12,71 años $\pm 0,11$, con una variabilidad promedio de 1,08 años, una edad mínima de 11 años, una máxima de 16 años y un coeficiente de variación de 8\%. Según los intervalos propuestos en la distribución fueron más frecuentes los adolescentes entre 11 y 13 años con un 69\%. En cuanto al sexo; el femenino predominó con un 66\% (33 Hembras), mientras que el masculino representó un 14\% (17 varones) de la muestra.

En lo que respecta al año de estudio los alumnos del 8 vo grado representaron un $36 \%$, los del 7 mo grado un $24 \%$ y los del 9 no grado un $20 \%$. Mientras que 4 to año representaron un $12 \%$ de la muestra y 5 to año un $8 \%$. El grado predominante fue el de 8 vo y $7 \mathrm{mo}$ grado, donde las investigadores detectaron el acoso por Bullying en un $10 \%$, lo que significa 5 estudiantes entre ambos grados.

En relación con las implicaciones de la dinámica Bullying, 28 alumnos fueron identificados por sus compañeros como involucrados en Bullying, representando un $56 \%$ de la muestra general y quedan distribuidos de la siguiente manera: 11 alumnos fueron identificados como fuertes (40\%); 4 alumnos además de fuertes son provocadores (14\%); 3 alumnos son clasificados provocadores y Agresivo (11\%); 2 alumno es Agresivo y Provocador (7\%). Igualmente, 3 alumnos sus compañeros manifiestan tenerle idea (11\%), 3 alumnos son considerados por sus compañeros como cobarde (11\%); 1 alumno es visto como cobardes-víctimas (3\%),) y un alumno que además de víctima es catalogada como provocador (3\%). (Tabla 1). 
Revista Interdisciplinaria de Humanidades, Educación, Ciencia y Tecnología

Año V. Vol. V. №9. Julio - Diciembre 2019

Hecho el depósito de ley: pp201602FA4721

ISSN-L: 2542-3029; ISSN: 2610-802X

Universidad Nacional Experimental Francisco de Miranda (UNEFM). Santa Ana de Coro. Venezuela

Ana Jiménez; Doralys Silva; Noheli Ortiz; Marinaisabel Vargas

\section{Tabla 1}

Adolescentes involucrados en la dinámica bullying, del liceo María Teresa Angulo. Sanare Edo Lara durante el periodo Enero-Junio 2017

\begin{tabular}{lcc} 
& FA & FR \\
\hline Fuertes & 11 & 40 \\
Fuertes/provocadores & 4 & 14 \\
Provocador/agresivo & 3 & 11 \\
Agresivo/provocador & 2 & 7 \\
Tenerle idea & 3 & 11 \\
Cobarde & 3 & 11 \\
Cobarde/victima & 1 & 3 \\
Victima/provocador & 1 & 3 \\
TOTAL & 28 & $100 \%$
\end{tabular}

Fuente: cuestionario para la exploración del Bullying (CEBU)

En relaciona al nivel de depresión en los estudiantes implicados en la dinámica Bullying, se encontró Ausente 50\% de la muestra estudiada (14 casos), en un 32\% (9 casos) presentan sintomatología Depresiva leve, mientras que un 11\% (3 casos) presentan depresión moderada y un 7\% (2 casos) presenta depresión severa. 


\section{Tabla 2}

Depresión

\section{CASOS}

\begin{tabular}{|c|c|c|}
\hline DEPRESIÓN & FA & FR \% \\
\hline Ausencia & 14 & 50 \\
\hline Leve & 9 & 32 \\
\hline Moderada & 3 & 11 \\
\hline Severa & 2 & 7 \\
\hline & 28 & 100 \\
\hline
\end{tabular}

Fuente: Instrumento de Beck

\section{DISCUSIÓN}

De acuerdo a los resultados arrojados por esta investigación, las investigadoras se afianza en los autores como Trautman, Skapinakis, Jansen y el mismo Olweus, que han destacado en el estudio de las consecuencias de ser agresor o víctima, e incluso ambos y su relación con la aparición de trastornos conductuales, disóciales que pueden llevar a actividad criminal, menor competitividad social, inseguridad y ansiedad, depresión e incluso ideación suicida $(2,10,20)$. De ahí la necesidad de realizar el estudio, porque a través de la observación directa de las investigadoras, y comprobada en el diagnóstico, se pudo evidenciar el acoso escolar entre pares por lo cual se hizo oportuno buscar solución a la problemática, con el fin de identificar como señalan los autores las consecuencias de ser agresor o víctima, e incluso ambos y su relación con la aparición de trastornos conductuales, disóciales, en este caso la depresión.

De los resultados obtenidos se presentó depresión severa en dos estudiantes, quienes pertenecen al $7 \mathrm{mo}$ y $8 \mathrm{vo}$ grado y predomina el sexo masculino entre las víctimas. Este resultado de la investigación guarda relación con el estudio de Díaz, Vanessa Daniella (2009) donde la causa más frecuente de maltrato entre iguales se encontró que el sexo 


\section{CIENCIAMATRIA \\ Revista Interdisciplinaria de Humanidades, Educación, Ciencia y Tecnología \\ Año V. Vol. V. №9. Julio - Diciembre 2019 \\ Hecho el depósito de ley: pp201602FA4721 \\ ISSN-L: 2542-3029; ISSN: 2610-802X \\ Universidad Nacional Experimental Francisco de Miranda (UNEFM). Santa Ana de Coro. Venezuela}

Ana Jiménez; Doralys Silva; Noheli Ortiz; Marinaisabel Vargas

predominante fue masculino. Con respecto a los protagonistas de este estudio resultó un total de 129 agresores, que prevaleció de 16-17 años (69,8\%), y en el presente estudio hubo 28 alumnos identificados por sus compañeros como involucrados en el caso de Bullying, representando esto un $56 \%$ de la muestra general.

Por otra parte, Monelos (2015) en su estudio empírico desde un punto de vista descriptivo y exploratorio, con la imposibilidad de acceder a una muestra aleatoria optó por un muestreo intencional y analizó el Bullying entendido como una forma específica de violencia escolar, donde encontró que uno o varios agresores con mayor poder y con intencionalidad de causar dolor acosan y someten reiteradamente a un compañero/a, tal como ha quedado de manifiesto. Este resultado se puede comparar con los datos obtenidos de los entrevistados quienes señalaron que cinco de sus compañeros son los más agredidos. De igual manera, el estudio les permitió a las investigadoras tomar las medidas de precaución y prevención cuando se les comunique a los padres y representante en las reuniones pautadas como ocurre el acoso escolar en el colegio y la actitud que toman algunos estudiantes al no comunicar la situación de acoso escolar, al profesorado y tampoco lo comunica a la familia.

Así mismo, la investigación de Acosta Rubí y Moreno Lismayka (2015) fue un aporte significativo para la presente investigación, porque muestra que las estrategias preventivas en contra del Bullying combinan las actuaciones dirigidas a mejorar las relaciones interpersonales a través de charlas, foros, eventos especiales, para mejorar el diálogo en todas sus formas bien sea con los compañeros de clase, con el ámbito social y la familia, a través de la cooperación, de la atención, de la igualdad.

\section{CONCLUSIONES}

La edad promedio fue de 12,71 años $\pm 0,11$, con una variabilidad promedio de 1,08 años, una edad mínima de 11 años, una máxima de 16 años y un coeficiente de 


\section{CIENCIAMATRIA \\ Revista Interdisciplinaria de Humanidades, Educación, Ciencia y Tecnología \\ Año V. Vol. V. №9. Julio - Diciembre 2019 \\ Hecho el depósito de ley: pp201602FA4721 \\ ISSN-L: 2542-3029; ISSN: 2610-802X \\ Universidad Nacional Experimental Francisco de Miranda (UNEFM). Santa Ana de Coro. Venezuela}

Ana Jiménez; Doralys Silva; Noheli Ortiz; Marinaisabel Vargas

variación de 8\% (serie homogénea entre sus datos). Según los intervalos propuestos en la distribución fueron más frecuentes los adolescentes entre 11 y 13 años con un 69\%.

En cuanto al sexo; el femenino predominó con un 66\% (33 Hembras), mientras que el masculino representó un 14\% (17 varones). También se obtuvo el grado de escolaridad donde los alumnos del 8 vo grado representaron un $36 \%$, los del 7 mo grado un $24 \%$ y los del 9 no grado un $20 \%$. Mientras que 4 to año representaron un $12 \%$ de la muestra y 5 to año un $8 \%$. El grado predominante fue el de $8 \mathrm{vo}$ y $7 \mathrm{mo}$ grado, donde las investigadores detectaron el acoso por Bullying en un 10\%, lo que significa 5 estudiantes entre ambos grados.

Con respecto a los adolescentes involucrados en la dinámica Bullying se obtuvo que 28 alumnos fueron identificados por sus compañeros como involucrados en el caso de Bullying, representando un $56 \%$ de la muestra general.

En líneas generales la sintomatología depresiva de los 28 alumnos, se presentó Ausente $50 \%$ de la muestra estudiada, un $32 \%$ presento sintomatología depresiva no incapacitante un $11 \%$ presentaron depresión moderada y un 7\% presento depresión severa.

\section{RECOMENDACIONES}

Hacer programas que permitan prevenir y frenar la frecuencia de intimidación y violencia en los planteles.

Crear campaña en cadenas televisadas y de radio promoviendo escuelas libres de Bullying.

A la institución en estudio tomar las medidas necesarias e informar a las instituciones de salud estadal y local, a los padres y representantes de los resultados obtenidos de la investigación, con la finalidad de mejorar la calidad de atención a los y las adolescentes. Capacitar al personal docente, en cuanto al tipo de intervención, que 


\section{CIENCIAMATRIA \\ Revista Interdisciplinaria de Humanidades, Educación, Ciencia y Tecnología \\ Año V. Vol. V. No9. Julio - Diciembre 2019 \\ Hecho el depósito de ley: pp201602FA4721 \\ ISSN-L: 2542-3029; ISSN: 2610-802X \\ Universidad Nacional Experimental Francisco de Miranda (UNEFM). Santa Ana de Coro. Venezuela}

Ana Jiménez; Doralys Silva; Noheli Ortiz; Marinaisabel Vargas

deben asumir con programas antibullying que puedan ser aplicados desde el ámbito individual, con sistemas de mediación entre pares y en la totalidad del centro escolar.

Planificar reuniones continuas con los representantes para informarles sobre las medidas preventivas que deben seguir para contrarrestar estos antivalores existente en la institución.

\section{REFERENCIAS CONSULTADAS}

1. Acosta Rubí y Moreno Lismayka (2015). Estrategias de Convivencia Escolar para la Prevención del Bullying en la Escuela Técnica "Los Magallanes" del Municipio San Diego Del estado Carabobo. Facultad de Ciencias de la Educación, de la Universidad de Carabobo, requisito para la optar al Título de Licenciada en Educación, Mención Integral titulado. Recuperado de http://www.riuc.bc.uc.edu.ve/bitstream/123456789/2793/3/9609.pdf

2. Albores-Gallo L, Sauceda JM, Ruiz S, Roque E. El acoso escolar (bullying) y su asociación con trastornos psiquiátricos en una muestra de escolares en México. Salud Pública de México 2011 Jun; 53: 220-227.

3. Agencia PANA - CECODAP. Informe Somos Noticias 2011; 20.

4. Amemiya I, Oliveros M, Barrientos F. Factores de riesgo de violencia escolar (bullying) severa en colegios privados de tres zonas de la sierra del Perú. AnFacMed 2009; 70: 255-258.

5. Bond L, Carlin JB, Thomas L, Rubin K, Patton G. Does bullying cause emotional problems? A prospective study of young teenagers.BMJ 2001 Sep 1; 323(7311):480-416 Arsenault L, Fisher H, Moffitt T, Caspi A.La intimidación y victimización como riesgo de autolesiones en la adolescencia temprana: estudio de cohorte longitudinal. BMJ 2012; 344: E2683.

6. Comisión Nacional de los Derechos Humanos (2016) Informe Anual de Actividades 2016 Descripción de las acciones realizadas por este Organismo para la divulgación, protección y defensa de los derechos humanos en México.

7. Davidson RJ, Putnam KM, Larson CL. Dysfunction in theneural circuity of 


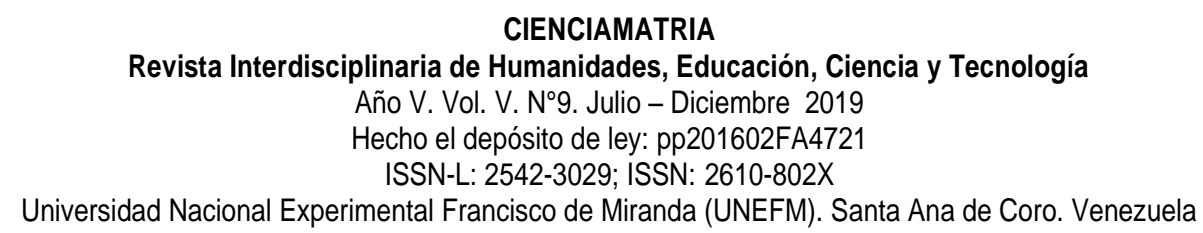

Ana Jiménez; Doralys Silva; Noheli Ortiz; Marinaisabel Vargas

emotion regulation a possible prelude to violence. Science 2000; 289: 591-4.

8. Dan Olweus (1995), Gewalt in der Schule. Was Lehrer und Eltern wissen sollten und tun können. Huber, Bern.

9. Díaz, Vanessa Daniella 2009 "Sindrome Bullying en estudiantes del ciclo diversificado del Liceo Bolivariano "Fernando Peñalver" de Ciudad Bolivar. Abril Julio.

10. García X, Pérez A, Nebot M. Factores relacionados con el acoso escolar (bullying) en los adolescentes de Barcelona. Gac Sanit 2010; 24(2):103-108.

11. Galdo, Redondo y García (2008). Atención al adolescente. Cantabria: Santand 32 Oñerreda, J. (2008). Bullying: concepto, causas, consecuencias, teorías y estudios epidemiológicos. XXVII Cursos de Verano EHU - UPV. Donostia - San Sebastián.19. Novo, J. (2011). Fisterrasalud. Recuperado el 26 de junio de 2011, http://www.fisterra.com/salud/1infoconse/depresion.asp.

12. Gini G, Puzzoli T. Asocciation betwen bullying and psychosomatic problems; a metanalysis. Pediatrics 2009; (123): 1059-1065.

13. Gómez Nashiki, A. (2013) Bullying: El poder de la violencia. Una perspectiva cualitativa sobre acosadores y víctimas en escuelas primarias de Colima Revista mexicana de investigación educativa Versión impresa ISSN 1405-6666 RMIE vol.18 no.58 México jul./sep. 2013.

14. Jansen D, Veenstra R, Ormel J, Verhulst FC. \&, Reijneveld SA.Early risk factors for being abully - victim, or bully /victim in late elementary and early secondary education. Thelongitudinal TRAILS study. BMC Public Health 2011; 6,11- 440.

15. McDowell, J. y Hostetler, B. (2000). Manual para consejeros de jóvenes. Alabama, EEUU:Editorial Mundo Hispano.

16. Monelos Muñiz $M^{a}$ Estrella (2015). Análisis de situaciones de acoso escolar o bullying en centros de Educación Secundaria de La Coruña Autor/a: Tesis de doctorante UDC / Universidad de Coruña. Recuperado de http://ruc.udc.es/dspace/bitstream/handle/2183/16155/MonelosMuniz MariaEstre lla TD 2015.pdf?sequence $=4$. 
17. Mejía Olivo Presenta: José Rafael (2011) El Bullying: el caso de la facultad de Contaduría y Administración de la Universidad Veracruzana región Xalapa T E S I S para obtener el Título de: Licenciado en Administración. Recuperado de http://cdigital.uv.mx/bitstream/123456789/32103/1/mejiaolivojoserafael.pdf.

18. Meza' Yenny Miranda Ana, Tejera Cesimar, Neidymar Tejera, Carmen Toloza (2015) Síndrome de bullying en estudiantes del Liceo José Luis Ramos. Maracay, Estado Aragua. Venezuela. Unidad de Investigación y Estudios en Salud Pública. Facultad de Ciencias de La Salud- Sede Aragua. Universidad de Carabobo http://www.scielo.org.ve/scielo.php?script=sci_arttext\&pid=S169032932015000200006.

19. Novo, J. (2011). Fisterrasalud. Recuperado de http://www.fisterra.com/salud/1infoconse/depresion.asp.

20. Organización Mundial de la Salud (OMS) (2010), Salud y Violencia en el Mundo, Informe mundial sobre la violencia y la salud: resumen, Publicado en español por la Organización Panamericana de la Salud para la Organización Mundial de la Salud. Washington, D.C..

21. Olweus D, Smith Pk, Morita $Y$, Jungers J. The nature of school bullying; a crossnatural perspective. 1st ed. London (UK): Routledge 2009. p. 31.

22. Pardo, G., Sandoval, A. y Umbarila D. (2008) Adolescencia y Depresión. Revista Colombiana de Psicología. Universidad nacional de Colombia. No. 13, 2004, pp. 13- Recuperado de http://scholar.google.es/scholar_url?hl=es\&q=http://dialnet.unirioja.es/servlet/artic ulo\%3Fcodigo\%3D3246301\%26orden\%3D259882\%26info\%3Dlink\&sa=X\&scisig $=$ AAGBfm0lbv4kCITXuyfSBk7QHKwzz20PMg\&oi=scholarr\&ei=mps_Ub6ZO4fV2 QX9 hYFg\&sqi=2\&ved=0CC4QgAMoAjAA.

23. Ponce Malaver Moisés (2015) Desarrollo Normal Del Adolescente Escula del Ministerio Publico Gerente Académico Lima,

24. Pittet I, Berchtold A, Akré C, Michaud PA, Surís JC. Are adolescents with chronic conditions particularly at risk for bullying? Arch Dis Child 2010;95:711-716.

25. Pierre M. El desarrollo cognitivo del niño: desde los descubrimientos de Piaget hasta las investigaciones actuales. Universidad de La Rioja. Contextos educativos 4(2001), 53-77. 


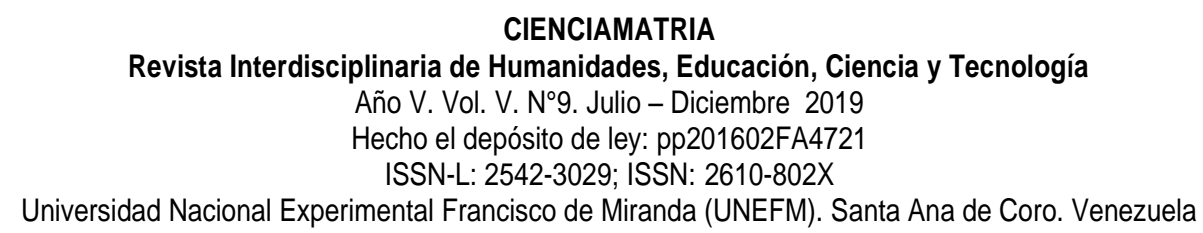

Ana Jiménez; Doralys Silva; Noheli Ortiz; Marinaisabel Vargas

26. Retamal, P. (2009). Depresión. Santiago, Chile: Editorial Universitaria.

27. Romani F, Gutiérrez C. Auto reporte de victimización escolar y factores asociados en escolares peruanos de educación secundaria. Rev Perú Epidemiol 2010; 14 (3).

28. Srabstein J, Leventhal B. Prevention of bullying - related morbidity and mortality: a call for public health policies. WorldHealthOrganization 2010; 88:403- 403.

29. Srabstein J, Pizarra T. Public Health, safety and educational risks asocciated with bullying behaviors in American Adolescents. Int J Adolesc Med Health 2008; (20):223-233.

30.Skapinakis, P., Bellos, S., Gkatsa. T., Magklara, K., Lewis, G., Araya, R., Stylianidis, S. \& Mavreas, V.The association between bullying and early stages of suicidal ideation in lateadolescents in Greece. BMC Psychiatry 2011; 8, 11-22.

31. Trautman A. Maltrato entre pares o "bullying"; una visión actual. Rev Chil Pediatr $2008 ; 79(1) ; 13-20$.

32. Vervoort $\mathrm{MH}$, Scholte $\mathrm{RH}$, Overbeek G. Bullying and victimization among adolescents: the role of ethnicity and ethnic composition of school class. J Youth Adolesc 2010; 39:1-11.

33. Vaughn MG, Fu Q, Bender K, Delisi M, Beaver KM, Perron BE, et al. Psychiatric Correlates of Bullying in the United States: Findings from National Sample. Psychiatr Q 2012;81: 183-195.

C2019 por los autores. Este artículo es de acceso abierto y distribuido según los términos y condiciones de la licencia Creative Commons Atribución-NoComercial-Compartirlgual 4.0 Internacional (CC BY-NC-SA 4.0) (https://creativecommons.org/licenses/by-nc-sa/4.0/). 